\section{Zoledronat adjuvant auch langfristig effektiv}

\author{
Schon die 48-Monats-Daten der "Austrian Breast and Colorectal Cancer Study \\ Group trial-12" (ABCSG-12) zeigten, dass die Ergänzung der adjuvanten \\ Therapie um Zoledronat das krankheitsfreie Überleben im Frühstadium \\ verlängert. Nun liegen die Ergebnisse für das weitere Follow-up vor.
}

Datier atientinnen, die vor der Menopause an Hormonrezeptor-positivem Brustkrebs in den Stadien I und II erkranken, bleiben länger erkrankungsfrei, wenn ihre Hormontherapie mit Tamoxifen oder Anastrozol um Zoledronat (4 mg alle sechs Monate) ergänzt wird. Laut ABCSG-12-Studie zum Follow-up nach median 62 Monaten reduziert Bisphosphonat das Rückfallrisiko um 32\% (Hazard Ratio [HR] 0,68; p = 0,009 ). Während $92 \%$ der Frauen unter Zoledronat keinen Rückfall erlitten hatten, waren es bei den ausschließlich endokrin behandelten $88 \%$. Alle Frauen hatten zudem Goserelin erhalten.

Bei Zugabe von Zoledronat zu Tamoxifen fiel die Risikoreduktion zwar ähnlich hoch aus, doch das Ergebnis war nicht signifikant (HR 0,67; p = 0,067). Ähnliche Zahlen wurden für die Anastrozol-Therapie mit oder ohne Zoledronat ermittelt (HR 0,68; $\mathrm{p}=0,061$ ).

Insgesamt wurden die Befunde von 1.803 Frauen in vier Gruppen verglichen: Goserelin plus Anastrozol \pm Zoledronat, Goserelin plus Tamoxifen \pm Zoledronat. Die eigentliche Krebstherapie lag zum Zeitpunkt des Follow-up schon über zwei Jahre zurück. Die Mortalität war durch Zoledronat jedoch noch nicht signifikant beeinflusst worden (HR 0,67; p = 0,09). Es zeigte sich aber, dass unter Anastrozol das Gesamtüberleben schlechter ausfiel als unter Tamoxifen (HR 1,75; $\mathrm{p}=0,02$ ).

Laut Autoren sei daher zu erwägen, in der adjuvanten Therapie Goserelin und Tamoxifen mit Zoledronat zu kombinieren - bei Frauen, die prämenopausal an Hormonrezeptor-positivem Brustkrebs im Frühstadium mit niedrigem oder mäßig erhöhtem Risiko litten. Die Ergebnisse passen den Forschern zufolge zu der Annahme, dass Zoledronat das Mikromilieu von Krebszellen im Knochenmark verändert: diesen werde es so erschwert, zu überleben und Rückfälle auszulösen.

Beim ASCO 2011 wurden inzwischen erste Daten nach 76-monatigem Follow-up präsentiert. Dort wurde nun auch ein si- gnifikanter Einfluss von Zoledronat auf die Gesamtmortalität gezeigt. Die durch das Bisphosphonat erzielte Reduktion beträgt im Vergleich zur alleinigen endokrinen Therapie laut einer Mitteilung der ABCSGWissenschaftler $41 \%$. Besonders effektiv sei Zoledronat in der Gruppe der über 40-Jährigen - hier liege die Sterblichkeit sogar um $49 \%$ niedriger. Robert Bublak

Gnant M et al. Adjuvant endocrine therapy plus zoledronic acid in premenopausal women with early-stage breast cancer: 62-month follow-up from the ABCSG-12 randomised trial.

Lancet Oncol. 2011;12(7):631-41.

Kommentar von Dr. Würstlein: Bisphosphonate spielen seit Jahren eine Rolle in der Therapie des ossär metastasierten Mammakarzinoms zur Reduktion von skelettalen Ereignissen, Hyperkalzämie sowie Schmerzen und sollten auch bei Progress in der oralen oder intravenösen Applikation fortgesetzt werden (s. AGO-Empfehlung 2011). Ebenso unumstritten ist ihr Einsatz oder der von RANK-L-Antikörpern bei Tumortherapie-induziertem Knochenverlust (Prävention oder Therapie).

Die Empfehlungen zum adjuvanten Einsatz von Bisphosphonaten unterliegen einem Wandel je nach aktueller Studienlage, was umso relevanter ist, da es sich um einen OffLabel-Use in der adjuvanten Therapiesituation bei prä- und postmenopausalen Patientinnen handelt. 2010 war eine Prävention von Metastasen bei primärem Mammakarzinom in allen Subgruppen empfohlen, wie in der AGOEmpfehlung 2011 für Subgruppen spezifiziert. Vor allem die AZURE-Studie konnte anders als erwartet krankheitsfreies (DFS) und Gesamtüberleben bei prämenopausalen Patientinnen nicht verbessern [Coleman RE et al. SABCS 2010; Abstr. S4-5]. Gegenüber der ABCSG-12Studie zeigen sich jedoch Unterschiede im Risikoprofil der Patientinnen (für ABCSG-12 war eine neoadjuvante Chemotherapie kein Ausschlusskriterium). Auch in der AZUREStudie konnte in der Subgruppe der mehr als fünf Jahre postmenopausalen Patientin eine
Risikoreduktion belegt werden. Umso wichtiger sind die jetzt aus der ABCSG-12-Studie präsentierten Daten mit medianem Follow-up von 62 Monaten. Interessanterweise wird der Effekt auf das DFS nicht durch die Wahl der antihormonellen Therapie (Tamoxifen oder Aromatase-Inhibitor), sondern durch die Zugabe des Bisphosphonats bestimmt - auch über die Zeit der adjuvanten Therapie im Follow-up hinaus. Dabei reduzierten sich sowohl ossäre als auch extraossäre Metastasen, ebenso waren Lokalrezidive und kontralaterale Mammakarzinome signifikant seltener unter bzw. nach Ende der BisphosphonatTherapie. Auch in der ABCSG-12-Studie zeigte

„Die Empfehlungen zum adjuvanten Einsatz von Bisphosphonaten unterliegen einem kontinuierlichen Wandel.”

sich dieser Effekt altersabhängig erst nach dem 40. Lebensjahr (Zeitpunkt der Ersterkrankung). Eine mögliche Erklärung ist die altersoder östrogenabhängige Beeinflussung des Knochenmilieus; Bisphosphonate könnten Trigger beim Auftreten von disseminierten Tumorzellen im Knochen sein.

Die häufigsten Nebenwirkungen unter Bisphosphonat waren Knochen-, Gelenk- und Kopfschmerzen, Arthralgie, Fatigue, Schlafstörungen und Fieber. In der Studie wurde kein Fall von Niereninsuffizienz oder Kieferosteonekrose beobachtet, was die Sicherheitsdaten zum adjuvanten Einsatz stärkt.

Die in Deutschland durchgeführten SOFTund TEXT-Studien lassen weitere Erkenntnisse erwarten. Die AGO-Empfehlung 2011 greift die aktuelle Datenlage mit einem ${ }_{\text {" }}+{ }^{\text {" }}$ zur adjuvanten Bisphosphonat-Gabe bei der prämenopausal endokrin therapierten Patientin unter gleichzeitiger Gabe von GnRH-Analoga sowie bei der postmenopausalen Patientin auf. Zur Therapie von Tumortherapie-induziertem Knochenmasseverlust/Osteoporose sollte ferner regelmäßig die Knochendichte bestimmt werden, außerdem sollten im Nachsorgegespräch Sport, körperliche Aktivität, Vitamin-D- und Kalzium-Einnahme sowie die Vermeidung eines zu niedrigen BMl empfohlen werden $\left({ }_{1}++{ }^{\prime \prime}\right)$.

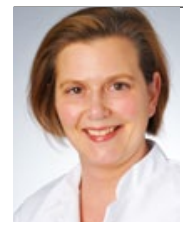

Dr. med. Rachel Würstlein Brustzentrum und Frauenklinik der Universität München rachel.wuerstlein@med. uni-muenchen.de 